\title{
RENDIMIENTO Y CALIDAD NUTRIMENTAL DE FRIJOL EJOTERO (Phaseolus vulgaris L.) EN FECHAS DE SIEMBRA
}

\author{
YIELD AND NUTRIMENTAL QUALITY OF SNAP BEAN (Phaseolus vulgaris L.) THROUGH \\ PLANTING DATES
}

\author{
Nicolás Salinas Ramírez ${ }^{1}$, J. Alberto Escalante Estrada ${ }^{1 *}$, Ma. Teresa Rodríguez González ${ }^{1}$ y \\ Eliseo Sosa Montes
}

\begin{abstract}
${ }^{1}$ Postgrado en Botánica, Colegio de Postgraduados-Campus Montecillo. Km 36.5 carr. México-Texcoco. 56230, Montecillo, Texcoco, Edo. de México. Tel. 01(595) 9520200 ext.1330. ${ }^{2}$ Departamento de Zootecnia, Universidad Autónoma Chapingo. 56230, Chapingo, Edo. de México.

*Autor para correspondencia (jasee@colpos.mx)
\end{abstract}

\section{RESUMEN}

En México el consumo per capita de frijol ejotero (Phaseolus vulgaris L.) ha aumentado de 0.9 a $1.1 \mathrm{~kg}$ en los últimos años, por lo que se requiere incrementar su productividad y su calidad. Ello puede lograrse mediante prácticas agronómicas. La fecha de siembra es útil, para aprovechar al máximo las características del clima y que el cultivo exprese su potencial productivo. El presente estudio se realizó en Montecillo, Estado de México bajo condiciones de temporal o secano durante el ciclo primavera verano del 2005. Se utilizó el cv. de frijol ejotero 'Hav-14' de crecimiento indeterminado trepador, y el girasol (Helianthus annuus L.) cv. 'Victoria' como espaldera viva. Se determinó el rendimiento y la calidad nutricional del ejote producido en cinco fechas de siembra: 2 y 17 de mayo, 1 y 16 de junio, y 1 de julio. Las variables ambientales registradas fueron: temperaturas máxima y mínima, radiación fotosintéticamente activa, lluvia, evaporación y fotoperíodo. El mayor rendimiento $\left(1170 \mathrm{~g} \mathrm{~m}^{-2}\right)$ se obtuvo de la siembra del 2 de mayo y el menor $\left(540 \mathrm{~g} \mathrm{~m}^{-2}\right)$ en la del 1 de julio. El ejote fresco mostró los valores más altos en cenizas $(9 \%)$, fósforo $(0.13 \%)$, fibra detergente ácido (22 \%), lignina (8.3 \%), proteína $(28.9 \%)$ y grasa $(0.88 \%)$ en la siembra temprana del 2 mayo; en la siembra tardía del 1 de julio observaron los valores más altos en calcio (1.8\%), carbohidratos solubles (47 \%), fibra detergente neutro $(27.7 \%)$, hemicelulosa $(12.3 \%)$ y materia orgánica (93.8 \%). La conservación del ejote congelado y al vacío por seis meses no afectó su contenido de proteína.

Palabras clave: Phaseolus vulgaris, calidad nutricional, fechas de siembra, rendimiento.

\section{SUMMARY}

In México the per capita consumption of snap bean (Phaseolus vulgaris $\mathrm{L}$.) has increased from 0.9 to $1.1 \mathrm{~kg}$ during the last years, thus making necessary to increase its productivity and quality; this can be achieved through agronomic practices. An optimal sowing time is important since it allows for taking advantaje of environmental conditions so that a cultivar may express its yield potential and nutritional quality. This study was carried out at Montecillo, State of México during 2005, under rainfed conditions. The snap bean cv. 'Hav-14' of indeterminate climbing growth habit and sun- flower (Helianthus annuus L.) cultivar 'Victoria' as living trellis, were used. The fresh pod yield and its nutritional quality in five sowing dates (May 2, May 17, June 1, June16 and July 1) were evaluated. The registered climate variables were: maximum and minimum temperatures, photosynthetic active radiation, rain, evaporation and photoperiod. The highest yield $\left(1170 \mathrm{~g} \mathrm{~m}^{-2}\right)$ was obtained on May 2 and the lowest $\left(540 \mathrm{~g} \mathrm{~m}^{-2}\right)$ on July 1 . The fresh pods showed the highest values of: ashes $(9 \%)$, phosphorus $(0.13 \%)$, acid detergent fiber (22 \%), lignin (8.3\%), protein $(28.9 \%)$ and fat $(0.88$ $\%)$ on the first planting date. On July 1 date the highest values were reached for calcium $(1.8 \%)$, soluble carbohydrates $(47 \%)$, neutral detergent fiber $(27.7 \%)$, hemicellulose $(12.3 \%)$ and organic matter $(\mathbf{9 3 . 8} \%)$. The freezing conservation of fresh pods during six months did not diminish its protein content.

Index words: Phaseolus vulgaris, nutritional quality, sowing dates, pod yield.

\section{INTRODUCCIÓN}

El frijol ejotero (Phaseolus vulgaris L.) contribuye en la alimentación humana por su contenido de proteína, fibra, calcio y vitamina A (Bittenbender et al., 1984). Según Esquivel et al. (2006), el consumo per capita en México se ha incrementado en los últimos años de 0.9 a $1.1 \mathrm{~kg}$. Para atender la demanda de frijol ejotero es necesario aumentar la producción y mejorar su calidad nutricional (Silbernagel et al., 1991). El rendimiento promedio nacional de ejote es de $9.3 \mathrm{t} \mathrm{ha}^{-1}$ y los Estados de Puebla, Morelos y Sinaloa son los de más alto rendimiento (14, 11 y $10 \mathrm{t} \mathrm{ha}^{-1}$, respectivamente).

En Hidalgo y Estado de México se reporta el más bajo rendimiento con 7 y $5 \mathrm{t} \mathrm{ha}^{-1}$, respectivamente (SAGARPA, 2003). Estos bajos rendimientos posiblemente se deben a que el cultivar utilizado, el manejo del cultivo y la fecha de siembra no son los adecuados para la región de 
Texcoco, Edo. de México, por lo que se justifica identificar las variantes más apropiadas para una óptima expresión del rendimiento. Los cultivares de frijol ejotero más utilizados en México son: 'Black Valentine', 'Contender', 'Kentucky Wonder', 'Sure Crop Wax' y 'Tendergreen' (Álvarez y Baca, 1986). Algunas propuestas recientes incluyen al cultivar 'Hav-14' de hábito indeterminado trepador (tipo IV), que requiere un soporte para su crecimiento (Escalante y Kohashi, 1993) y que no está caracterizado para la región de Texcoco, como tampoco lo están las prácticas agronómicas apropiadas para que exprese su máxima producción y calidad (Peixoto et al., 2001). En el cultivo de frijol ejotero de crecimiento indeterminado comúnmente se utilizan como soporte estructuras de madera, metálicas y de concreto que incrementan el costo de producción; una alternativa para reducirlo es el uso de espaldera viva como el girasol (Helianthus annuus L.) cv. 'Victoria', que tiene características deseables, tallo erecto y raíz profunda, que le permiten un buen anclaje y ser soporte para el crecimiento de frijol de guía (Escalante, 1995).

El rendimiento de frijol varía en función del fotoperíodo (Acosta y White, 1995), la intensidad luminosa (Escalante et al., 2001) y temperatura ambiental (Suzuki et $a l ., 2001)$. Algunos efectos de la fecha de siembra en frijol ejotero en el Valle de México fueron reportados por Esquivel et al. (2006), quienes en dos fechas de siembra ( 21 de mayo y 15 de junio) compararon a 36 cultivares de frijol ejotero de los hábitos de crecimiento tipos I, III y IV; observaron que el rendimiento promedio más alto $\left(25.6 \mathrm{t} \mathrm{ha}^{-1}\right)$ correspondió a la siembra del 21 de mayo y el más bajo $\left(11.5 \mathrm{t} \mathrm{ha}^{-1}\right)$ a la del 15 de junio, diferencia que atribuyeron al cambio en la temperatura mínima y del fotoperíodo. Según Peixoto et al. (2002), el cultivar de frijol ejotero 'Hav-14' sembrado el 30 de abril en Anápolis, Brasil con clima cálido, mostró rendimientos que van desde 10.89 a 21.7 t ha $^{-1}$ en 1996 y 1998, respectivamente, con 42 d de siembra a inicio de floración. En Korea (clima templado) Lee et al. (2001) evaluaron fechas de siembra en frijol ejotero entre el 20 de marzo y el 18 de junio, y encontraron que la siembra del 4 de abril produjo $24 \mathrm{tha}^{-1}$ debido a la aplicación de riego.

La calidad nutricional (contenidos de carbohidratos y lípidos) en el cultivo de maíz (Zea mays L.) y girasol, varía entre fechas de siembra (Furano y Fernández, 2005). En contraste, el contenido de calcio en ejote no mostró diferencias significativas entre las siembras hechas del 15 de junio al 29 de junio, en las que se registraron precipitaciones de 134 y $246 \mathrm{~mm}$, con una acumulación de unidades calor de 1317 y 1535 durante el ciclo del cultivo, respectivamente (Quintana et al., 2001). Tendencias similares se observaron para el contenido de fibra entre cultivares de frijol ejotero de hábito de crecimiento indeterminado y determinado sembrados en Texcoco (clima templado) el 21 de mayo y 15 de junio (Esquivel et al., 2006). Álvarez y Baca (1986) compararon a los cvs. 'Contender', 'Tendergreen', 'Kentucky Wonder', 'Top Crop' y 'Strigless Black Valentine', y recomendaron las siguientes fechas de siembra: a) del 1 de abril al 30 de julio en la meseta central; b) del 1 de octubre al 15 de noviembre en el noreste del país ; c) del 1 de septiembre al 30 de enero en las llanuras de Veracruz; d) del 1 de marzo al 30 de julio para la zona de El Bajío.

Por su parte, Hernández (1999) señaló que el ejote presenta elevada tasa respiratoria y consecuentemente corta vida de anaquel (10 d, en promedio). Entre los métodos de conservación que preservan las características de calidad, la congelación ha tenido amplia aceptación, por las ventajas que ofrece: mínima pérdida del contenido nutricional y conservación del producto hasta por 12 meses. La congelación solidifica el agua contenida en las células y detiene así los procesos biológicos y enzimáticos que degradan los alimentos (Hernández, 1999).

Los nutrimentos que contiene el ejote cumplen funciones vitales en el organismo humano, como calcio y fósforo que son esenciales para la formación de huesos y dientes. Las personas adultas tienen un requerimiento diario que oscila entre 800 y $1200 \mathrm{mg}$ de ambos elementos (Quintana et al., 2001). Los carbohidratos solubles se consideran la principal fuente de energía para mantener la temperatura y para el movimiento corporal, y su requerimiento es de $5 \mathrm{~g} \mathrm{~kg}^{-1} \mathrm{~d}^{-1}$. La celulosa, hemicelulosa y lignina no son asimilables por el tracto digestivo pero reducen problemas de estreñimiento y de colesterol, regulan la digestión, limpian el intestino grueso y delgado; su requerimiento es de 20 a $40 \mathrm{~g} \mathrm{~d}^{-1}$. La proteína es necesaria para la formación de órganos, tejidos, sangre, piel, uñas, hormonas, etc., y su requerimiento diario es de $0.9 \mathrm{~g}$ por $\mathrm{kg}$ de peso corporal. Los lípidos sirven para amortiguar las bajas temperaturas, proporcionan $40 \%$ de la energía y facilitan la absorción de las vitaminas A, D, E y K; su requerimiento diario es de 0.5 a $1 \mathrm{~g} \mathrm{~kg}^{-1}$ (Johnt et al., 1999).

En este estudio se determinó el efecto de la fecha de siembra sobre el desarrollo, rendimiento y calidad nutricional del frijol ejotero cv. 'Hav-14', así como determinar si la congelación como método de conservación afecta el contenido de $\mathrm{Ca}$, $\mathrm{P}$ y proteína.

\section{MATERIALES Y MÉTODOS}

El estudio se hizo en Montecillo, Texcoco, Estado de México (19² $29^{\prime} \mathrm{LN}, 98^{\circ} 53^{\prime} \mathrm{LO}$ y $\left.2250 \mathrm{msnm}\right)$, con 
clima BS1 que es el menos seco de los áridos con lluvias en verano, temperatura media anual de $14.6{ }^{\circ} \mathrm{C}$ y 558.5 mm de precipitación anual (García, 2005). El suelo del sitio experimental es de textura arcillosa (Fluvisol mólico), con 2 a $3 \%$ de materia orgánica y $\mathrm{pH}$ de 8.0, en los primeros $30 \mathrm{~cm}$ de perfil. El material biológico de frijol ejotero fue el cultivar 'Hav-14' de hábito de crecimiento indeterminado trepador (Tipo IV; Singh, 1982) desarrollado por el programa de frijol del Centro Internacional de Agricultura Tropical de Cali, Colombia. Como soporte para la planta se utilizó el girasol cv. 'Victoria'. Los tratamientos consistieron en cinco fechas de siembra: 2 y 17 de mayo, 1 y 16 de junio y 1 de julio del 2005, todas en condiciones de temporal o secano, con un riego de presiembra para las dos primeras fechas. El experimento se fertilizó a los 30 d después de cada fecha de siembra con la dosis $100 \mathrm{~N}-100 \mathrm{P}-00 \mathrm{~K} \mathrm{~kg} \mathrm{ha}{ }^{-1}$. El diseño experimental fue de bloques completos al azar con cuatro repeticiones.

Durante el ciclo se registraron las temperaturas máxima y mínima $\left({ }^{\circ} \mathrm{C}\right)$, radiación fotosintéticamente activa $\left(\right.$ RFA, en $\left.\mathrm{MJ} \mathrm{m}^{-2}\right)$, precipitación pluvial $(\mathrm{mm})$, evaporación $(\mathrm{mm})$ y duración del fotoperíodo, con datos proporcionados por la estación agrometereológica del Colegio de Postgraduados. Las unidades calor (UC) acumuladas se calcularon con el método residual (Snyder, 1985), mediante la ecuación: UC $=\sum[($ Tmáx + Tmin $) / 2-T b]$; donde: Tmáx $=$ Temperatura máxima diaria $\left({ }^{\circ} \mathrm{C}\right) ;$ Tmin $=$ Temperatura mínima diaria $\left({ }^{\circ} \mathrm{C}\right) ; \mathrm{Tb}=$ Temperatura base o umbral $\left(10{ }^{\circ} \mathrm{C}\right.$, según Escalante et al., 2001). La evapotranspiración del cultivo (ETc, en $\mathrm{mm} \mathrm{d}^{-1}$ ) se estimó mediante la ecuación $\mathrm{ETc}=\mathrm{Ev} \times \mathrm{Ke} \times \mathrm{Kc}$, donde: $\mathrm{Ev}=$ Evaporación del tanque tipo $\mathrm{A} ; \mathrm{Ke}=$ Coeficiente para el evaporímetro (0.6); y Kc = Coeficiente del cultivo (0.65) (Doorenbos y Pruitt, 1986).

Las variables determinadas en planta fueron: la fenología (etapas vegetativas y reproductivas) del frijol ejotero, como señalaron Escalante y Kohashi (1993), y rendimiento total en fresco $\left(\mathrm{g} \mathrm{m}^{-2}\right)$ y número de ejotes por $\mathrm{m}^{2}$. Se efectuaron ocho cortes con intervalos de $3 \mathrm{~d}$ en los que se cosecharon sólo los ejotes con más de $10 \mathrm{~cm}$ de longitud. Se tomaron muestras de todos los tratamientos y se escaldaron por inmersión en agua a $98{ }^{\circ} \mathrm{C}$ durante $3 \mathrm{~min}$, seguida de una segunda inmersión a $4{ }^{\circ} \mathrm{C}$ durante $4 \mathrm{~min}$, se empacaron al vacío y conservaron a $-36{ }^{\circ} \mathrm{C}$ durante seis meses. Para evaluar la calidad nutrimental del ejote fresco y congelado, las muestras provenientes de las cinco fechas de siembra se desecaron a $55{ }^{\circ} \mathrm{C}$ en una estufa de aire forzado hasta peso constante $(3 \mathrm{~d})$. Posteriormente las muestras fueron molidas en un molino eléctrico con criba de $5 \mu \mathrm{m}$ para determinar: humedad, materia seca, materia orgánica, cenizas totales, calcio, fósforo, fibra detergente neutro (FDN), hemicelulosa, fibra detergente ácido (FDA), lignina, proteína cruda, grasa cruda, carbohidratos solubles y hemicelulosa (AOAC, 1975; Sosa, 1979).

\section{RESULTADOS Y DISCUSIÓN}

\section{Fenología y condiciones ambientales}

Las etapas fenologícas del cultivar 'Hav-14' mostraron cambios por efecto de la fecha de siembra. En la siembra del 1 de julio, en comparación con la del 2 de mayo, el ciclo del cultivo se redujo en $8 \mathrm{~d}$, la emergencia en $2 \mathrm{~d}$, el inicio de floración en $8 \mathrm{~d}$, y el primero y último corte de ejote en 6 y 8 d, respectivamente. Además, en la siembra del 1 de junio se observó que la iniciación de la primera hoja trifoliolada y prefloración presentaron diferencias en precocidad de 14 y $10 \mathrm{~d}$, con respecto a las otras fechas, pero dicho comportamiento y su relación con las variables ambientales no es clara (Figura 1). Tales respuestas de las siembra del 1 de junio se relacionaron con un descenso en la temperatura media máxima durante el ciclo de 38 a 32 ${ }^{\circ} \mathrm{C}$, ya que este factor influye en la duración de las etapas fenológicas (Masaya y White, 1991). Rosales et al. (2001) señalaron que a baja temperatura $\left(9\right.$ a $\left.10^{\circ} \mathrm{C}\right)$ e incremento en la precipitación, se retrasa la floración en el cultivo del frijol.

En la siembra del 2 de mayo se presentó la menor precipitación acumulada durante el ciclo con $263 \mathrm{~mm}$, en contraste con la siembra del 1 de julio donde se registró la más alta de $364 \mathrm{~mm}$ (Cuadro 1 y Figura 1). En la siembra del 2 de mayo ocurrió un déficit hídrico durante las etapas vegetativas, debido a que la evapotranspiración (ETc) superó a la precipitación (Figura 2). En la siembra del primero de julio (Figura 3), un déficit similar se registró en la etapa reproductiva, que influyó en forma negativa en el rendimiento.

\section{Unidades calor (UC) y evapotranspiración (ETc)}

Las UC y la ETc acumuladas durante el ciclo del cultivo difirieron entre fechas de siembra; sus valores más altos se observaron en la siembra del 2 de mayo $\left(1087^{\circ} \mathrm{C}\right.$ y $224 \mathrm{~mm}$ ) y disminuyeron conforme la siembra se hizo más tarde, de modo que los valores más bajos $\left(844^{\circ} \mathrm{C}\right.$ y $147 \mathrm{~mm}$ ) correspondieron a la siembra del 1 de julio (Cuadro 1). Esto sugiere una posible relación entre la producción de ejote y estos índices, lo cual requiere verificarse con estudios adicionales. 


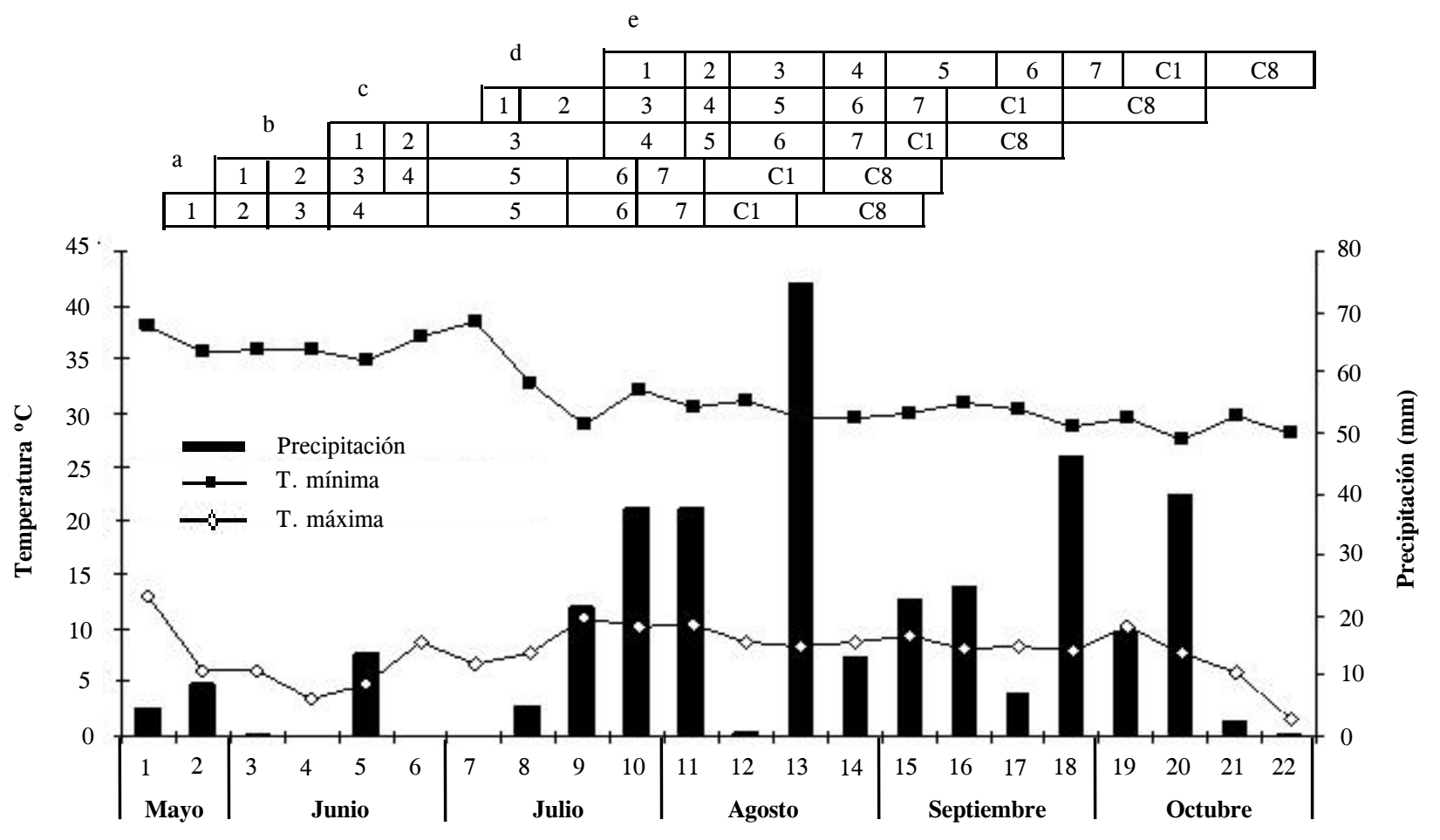

Semanas después de la siembra

Figura 1. Temperaturas máxima y mínima (medias semanales) y precipitación semanal en Montecillo, Texcoco, Estado de México. Verano 2005. Etapas fenológicas del frijol ejotero cv. 'Hav-14': 1 = Emergencia; 2 = Hojas primarias; 3 = Primera hoja trifoliolada; 4 = Tercera hoja trifoliolada; 5 = Prefloración; 6 = Floración; $7=$ Formación de vainas; $\mathrm{C} 1=$ Primer corte; $\mathbf{C 8}=$ Octavo corte. Fechas de siembra: $\mathbf{a}=\mathbf{2}$ de mayo, b $=$ 17 de mayo, $c=1$ de junio, $\mathrm{d}=16$ del junio, $\mathrm{e}=1 \mathrm{de}$ julio.

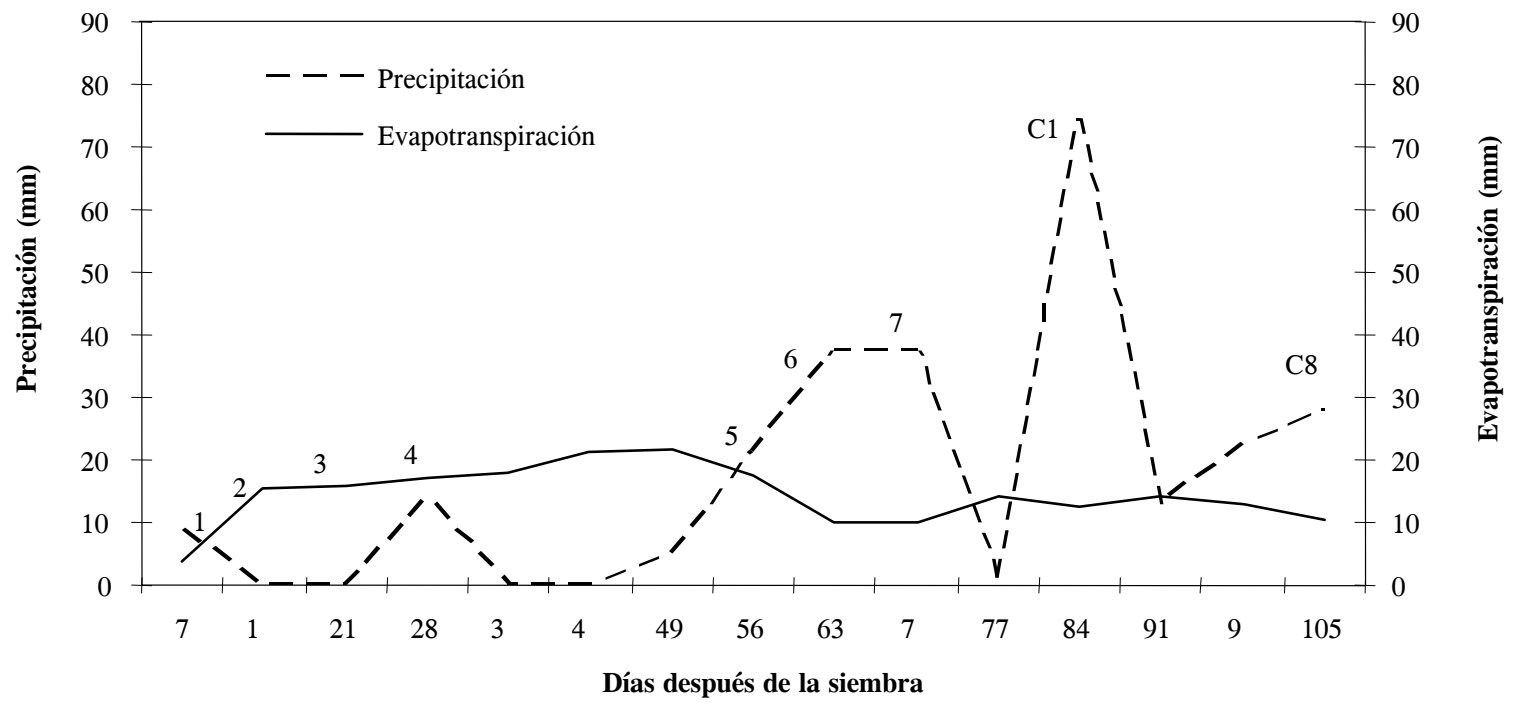

Figura 2. Dinámica de la precipitación y evapotranspiración durante el ciclo del cultivo de frijol ejotero 'Hav-14', en siembra del 2 de mayo. Montecillo, Texcoco, Estado de México. Verano 2005. 


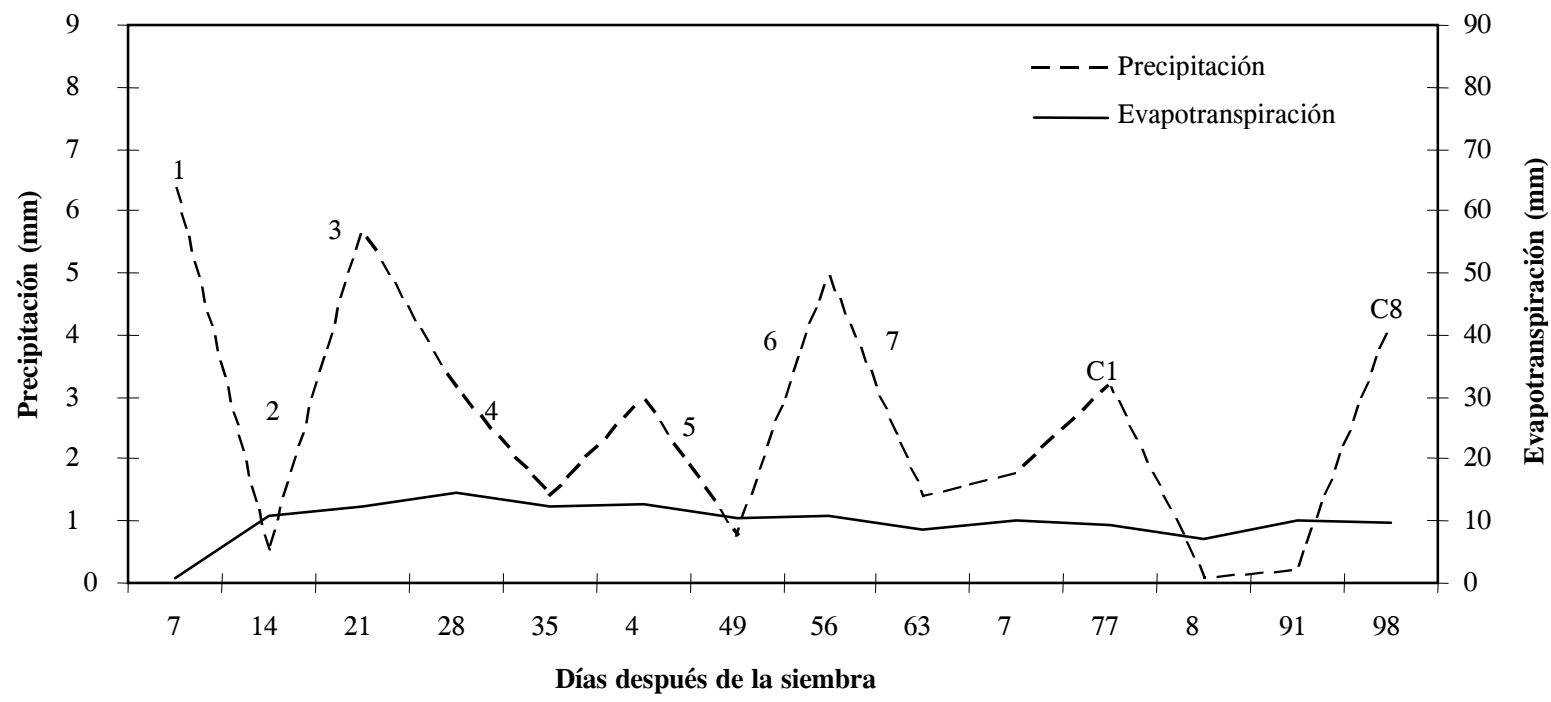

Figura 3. Dinámica de la precipitación y evapotranspiración, en el cultivo de frijol ejotero 'Hav-14', en la siembra del 1 de julio. Montecillo, Texcoco, México. Verano 2005. C1 a C8 se explican en la Figura 1.

Cuadro 1. Rendimiento y número de ejotes por unidad de área, para cada fecha de siembra del cv. 'Hav-14', unidades calor, evapotranspiración del cultivo, radiación, fotoperíodo y precipitación acumulados durante el ciclo del cultivo. Montecillo, Texcoco, Estado de México. 2005.

\begin{tabular}{lccccccc}
\hline $\begin{array}{l}\text { Fecha de } \\
\text { siembra }\end{array}$ & $\begin{array}{c}\text { Rendimiento } \\
\left(\mathrm{g} \mathrm{m}^{-2}\right)\end{array}$ & $\begin{array}{c}\text { Núm. ejotes } \\
(\text { ejotes m })^{-2}\end{array}$ & UC & $\begin{array}{c}\text { ETc } \\
(\mathrm{mm})\end{array}$ & $\begin{array}{c}\text { RFA } \\
\left(\mathrm{MJ} \mathrm{m}^{-2}\right)\end{array}$ & $\begin{array}{c}\text { Fotoperíodo } \\
\text { promedio }\left(\mathrm{h} \mathrm{d}^{-1}\right)\end{array}$ & $\begin{array}{c}\text { Precipitación } \\
\left(\mathrm{mm}^{2}\right)\end{array}$ \\
\hline 02 de mayo & $1170 \mathrm{a}$ & $258 \mathrm{a}$ & 1087 & 224 & 972 & 13.0 & 263 \\
17 de mayo & $1000 \mathrm{ab}$ & $189 \mathrm{ab}$ & 1048 & 207 & 956 & 12.9 & 317 \\
01 de junio & $930 \mathrm{ab}$ & $155 \mathrm{ab}$ & 940 & 176 & 926 & 12.9 & 294 \\
16 de junio & $660 \mathrm{ab}$ & $151 \mathrm{ab}$ & 881 & 148 & 836 & 348 \\
01 de julio & $540 \mathrm{~b}$ & $102 \mathrm{~b}$ & 844 & 147 & 791 & 12.6 \\
\hline
\end{tabular}

Medias con la misma letra dentro de columnas son estadísticamente iguales (Tukey 0.05).

$\mathrm{UC}=$ Unidades calor acumuladas; ETc $=$ Evapotranspiración acumulada; RFA = Radiación fotosintéticamente activa .

\section{Radiación fotosintéticamente activa (RFA) y fotoperíodo}

Entre fechas de siembra la RFA más alta correspondió a la del 2 de mayo con $972 \mathrm{MJ} \mathrm{m}^{-2}$ y su valor disminuyó en fechas posteriores; la siembra del 1 de julio presentó la más baja RFA acumulada (791 $\left.\mathrm{MJ} \mathrm{m}^{-2}\right)$. La variación del fotoperíodo durante el experimento fue mínima, con un rango de 12.6 a $13 \mathrm{~h}$ luz (Cuadro 1).

\section{Rendimiento y número de ejotes}

En la siembra del 2 de mayo se obtuvieron $1170 \mathrm{~g} \mathrm{~m}^{-2}$ y 258 ejotes $\mathrm{m}^{-2}$. En siembras posteriores los valores fueron menores, y los mínimos ocurrieron en la del $1 \mathrm{de}$ julio con $540 \mathrm{~g} \mathrm{~m}^{-2}$ y 102.5 ejotes por $\mathrm{m}^{2}$, respectivamente (Cuadro 1). No hubo diferencias estadísticas, pero sí numéricas de $500 \mathrm{~g} \mathrm{~m}^{-2}$, entre la primera y cuarta fecha de siembra. Estos resultados son similares a los encontrados por Pexioto et al. (2002) quienes reportaron un rendimiento de $1089 \mathrm{~g} \mathrm{~m}^{-2}$ para el cultivar 'Hav-14' con siembras de otoño a invierno, pero son inferiores a lo reporta- do por Esquivel et al. (2006) quienes obtuvieron $2560 \mathrm{~g}$ $\mathrm{m}^{-2}$ en variedades de ejote de crecimiento indeterminado, en condiciones del Valle de México bajo riego. En general, las diferencias en rendimiento observadas en este estudio se atribuyen a la variación en precipitación y temperatura que hubo durante el ciclo.

\section{Relaciones entre rendimiento e índices ambientales}

El modelo de regresión que mejor estimó el rendimiento en función de los índices ambientales fue $\mathrm{y}=$ $911.2+3.98 \mathrm{ETc}+1.19 \mathrm{RFA}$, con $\mathrm{R}^{2}=0.99$, lo que indica que por cada mm de ETc y por cada MJ $\mathrm{m}^{2}$ de RFA que incide sobre el dosel vegetal, se incrementa el rendimiento en 3.98 y 1.19 g, respectivamente. Estos resultados coinciden con los encontrados por Escalante $e t$ al. (2001) para el frijol de grano, quienes observaron que el rendimiento está determinado por la ETc y radiación solar acumulados durante el ciclo. Por su parte, Calvache et al. (1997) reportaron en Ecuador que el frijol de grano cv. 'Imbabello' sembrado el 3 de julio acumuló 1272 de UC y $445 \mathrm{~mm}$ de ETc, valores que son superiores a los 
reportados en el presente estudio para la misma fecha de siembra.

\section{Calidad de ejote}

En la siembra del 2 de mayo se obtuvieron los más altos en cenizas totales, fósforo, fibra detergente ácido, lignina, proteína y grasa; los valores más bajos correspondieron a la siembra del 1 de julio. En contraste, los contenidos más altos de calcio, carbohidratos solubles, fibra detergente neutro, hemicelulosa y materia orgánica, se encontraron en la siembra del 1 de julio. Quintana et al. (2001) no observaron diferencias significativas por efecto de la fecha de siembra (15 y 28 de junio), precipitación de 134 y 246 mm y UC de 1317 y 1535, respectivamente; según estos investigadores la acumulación de calcio en vaina estuvo influenciada por el cultivar y no por los cambios en el ambiente. El contenido de proteína aquí registrada (28.9 \%, Cuadro 2) fue similar al encontrado en otros cultivares de frijol ejotero de hábito de crecimiento indeterminado (Esquivel et al., 2006).

En cuanto a la calidad nutrimental del frijol ejotero se observó que $100 \mathrm{~g}$ de ejote fresco del cv. 'Hav-14' cosechados de la siembra del 2 de mayo, proporcionan $95 \mathrm{mg}$ de calcio, $11 \mathrm{mg}$ de fósforo, $3400 \mathrm{mg}$ de carbohidratos solubles, $4730 \mathrm{mg}$ de fibra, $2490 \mathrm{mg}$ de proteína y $76 \mathrm{mg}$ de grasa, mientras que con la siembra del 1 de julio los valores fueron $159 \mathrm{mg}$ de calcio, $11 \mathrm{mg}$ de fósforo, 4150 $\mathrm{mg}$ de carbohidratos, $5200 \mathrm{mg}$ de fibra, $1820 \mathrm{mg}$ de proteína y $49 \mathrm{mg}$ de grasa; es decir los ejotes procedentes de la siembra del 2 de mayo contienen más proteína, fósforo, fibra detergente ácido, lignina y grasa que los de la siembra 1 de julio en la que el mayor aporte fue en carbohidratos solubles y fibra. El consumo de $100 \mathrm{~g}$ de ejote de la siembra 2 de mayo cubre parte de las necesidades dia- rias de nutrimentos de una persona de $70 \mathrm{~kg}$ : $1 \%$ del fósforo, $10 \%$ del calcio, $16 \%$ de la fibra y $4 \%$ de la proteína. En congruencia con estos resultados, la fecha del 2 de mayo registró más UC acumuladas (1087) y mayor RFA (792 MJ m ${ }^{-2}$, Cuadro 1), que la del 1 de julio.

Por ser una hortaliza de corta vida de anaquel (10 d) el ejote se congela para preservar su calidad nutrimental (Hernández, 1999). La calidad del ejote cv. 'Hav-14' de la siembra del 1 de junio conservó el contenido de calcio, fósforo y proteína (Cuadro 3). Steinbuch (1980) reportó que la calidad evaluada a través del color, sabor y textura se mantuvo sin cambio bajo condiciones de almacenamiento similares a las del presente estudio.

\section{CONCLUSIONES}

La fenología, el rendimiento y la calidad nutrimental del frijol ejotero varían en función del clima que ocurre durante el ciclo del cultivo, el cual puede variar a través de fechas de siembra. El rendimiento más alto se logró con la siembra del 2 de mayo y el más bajo con la del 1 de julio. El rendimiento se relacionó con la acumulación de unidades calor, la evapotranspiración y la radiación fotosintéticamente activa. Con base en el contenido de calcio, fósforo y proteína, la congelación al vacío no disminuyó la calidad del ejote.

\section{AGRADECIMIENTOS}

Al Consejo Nacional de Ciencia y Tecnología (CONACYT) por el apoyo otorgado para la realización de esta investigación. Registro No. 193001 (2005). Al programa de Granos y Semillas de la UACH por proporcionar la semilla del cv. 'Hav-14'.

Cuadro 2. Análisis nutrimental (\%) de frijol ejotero cv. 'Hav-14' para dos fechas de siembra. Montecillo, Texcoco, Estado de México. 2005.

\begin{tabular}{lccccccccccc}
\hline F. siembra & Cenizas & Calcio & Fósforo & CS & FDN & Hemicelulosa & FDA & Lignina & Proteína & Grasa & MO \\
\hline mayo & $9.0 \mathrm{a}$ & $1.1 \mathrm{~b}$ & $0.138 \mathrm{a}$ & $39.7 \mathrm{~b}$ & $23.3 \mathrm{~b}$ & $1.3 \mathrm{~b}$ & $22 \mathrm{a}$ & $8.3 \mathrm{a}$ & $28.9 \mathrm{a}$ & $0.88 \mathrm{a}$ & $90.9 \mathrm{~b}$ \\
1 julio & $6.1 \mathrm{~b}$ & $1.8 \mathrm{a}$ & $0.133 \mathrm{~b}$ & $47.0 \mathrm{a}$ & $27.7 \mathrm{a}$ & $12.3 \mathrm{a}$ & $14.1 \mathrm{~b}$ & $0.5 \mathrm{~b}$ & $20.6 \mathrm{~b}$ & $0.56 \mathrm{~b}$ & $93.8 \mathrm{a}$ \\
\hline
\end{tabular}

Medias con la misma letra dentro de columnas son estadísticamente iguales (Tukey 0.05 ).

F. siembra $=$ Fecha de siembra; $\mathrm{CS}=$ Carbohidratos solubles; FDN = Fibra detergente neutro o paredes celulares; FDA = Fibra detergente ácido; MO

= Materia orgánica. La humedad del ejote fresco fue de $91 \%$ para la fecha 2 de mayo, y de $90.8 \%$ para la del 1 de junio.

Cuadro 3. Análisis nutrimental (en \% del peso seco) en frijol ejotero 'Hav-14' fresco y congelado. Montecillo, México. Verano 2005. Datos promedio de cuatro repeticiones.

\begin{tabular}{|c|c|c|c|c|c|c|c|c|c|c|c|}
\hline Tratamientos & Cenizas & Calcio & Fósforo & $\mathrm{CS}$ & FDN & Hemicelulosa & FDA & Lignina & Proteína & Grasa & MO \\
\hline Congelado & $7.4 \mathrm{a}$ & $1.6 \mathrm{a}$ & $0.02 \mathrm{a}$ & $43.9 \mathrm{a}$ & $25.1 \mathrm{~b}$ & $7.6 \mathrm{~b}$ & $17.4 \mathrm{a}$ & $0.2 \mathrm{~b}$ & $20 \mathrm{a}$ & $3.4 \mathrm{a}$ & $92.5 \mathrm{a}$ \\
\hline Fresco & $7.1 \mathrm{a}$ & $1.6 \mathrm{a}$ & $0.14 \mathrm{a}$ & $37.7 \mathrm{~b}$ & $29.1 \mathrm{a}$ & $13.3 \mathrm{a}$ & $15.7 \mathrm{~b}$ & $0.4 \mathrm{a}$ & $24.3 \mathrm{a}$ & $0.6 \mathrm{a}$ & $92.8 \mathrm{a}$ \\
\hline
\end{tabular}

Medias con la misma letra dentro de columnas son estadísticamente iguales (Tukey 0.05).

$\mathrm{CS}=$ Carbohidratos solubles; FDN $=$ Fibra detergente neutro o paredes celulares; FDA $=$ Fibra detergente ácido; MO = Materia orgánica. La humedad del ejote fresco fue de $90.7 \%$ para la fecha 1 de junio. 


\section{BIBLIOGRAFÍA}

Acosta-Gallegos J A, J White W (1995) Phenological plasticity as an adaptation by common bean to rainfed environments. Crop Sci. 35:199-204.

Álvarez E L, G Baca C (1986) El Frijol Ejotero. Servicio de Extensión Agropecuaria. México,D. F. 46 p.

AOAC (1975) Oficial Methods of Analysis of the Association of official Analytical Chemists. 12th edition. Washington, USA. 354 p.

Bittenberder H C, R Barret P, B Indere - Larusa (1984) Beans and cowpeas as leaf vegetables and grain legumes. Bean Cowpea Collaborative Research Support Program. Monograph no.1. Michigan State University. East Lansing, MI, USA. 24 p.

Calvache M, K Reichardt, O O Bacchi S, D Neto D (1997) Deficit irrigation at different growth stages of the common bean (Phaseolus vulgaris L.) cv. Imbabello. Sci. Agric. Brasil. 54:103-119.

Doorenbos J, W Pruitt O (1986) Las Necesidades del Agua para los Cultivos. Estudio FAO. Riego y Drenaje. Manual 24. 194 p.

Escalante E J A (1995) Aprovechamiento del recurso agua en cultivos de secano. Agroproductividad 12:28-32.

Escalante E J A, J Kohashi S (1993) El Rendimiento y Crecimiento del Frijol. Manual para Toma de Datos. Colegio de Postgraduados. México. 84 p.

Escalante E J A, L E Escalante E, M T Rodríguez G (2001) Producción de frijol en dos épocas de siembra: Su relación con la evapotranspiración, unidades calor y radiación solar en clima cálido. Terra 19:309-315.

Esquivel E G, A Acosta-Gallegos J, R Rosales S, P Pérez H, M J Hernández C, R Navarrete M, S J Muruaga M (2006) Productividad y adaptación de frijol ejotero en el valle de México. Rev. Chapingo S. Hort. 12:109-116.

Furano D, P Fernández J (2005) Rendimiento potencial y fechas de siembra para maíz y girasol. Cosecha Gruesa 16:24-27.

García E L (2005) Modificación al Sistema de Clasificación Climática de Köppen. 4a ed. UNAM. México, D.F. 217 p.

Hernández V F (1999) Conservas Caseras de Alimentos. 3a ed. Ed. Mundi-Prensa. Madrid, España. 198 p.

Johnt T R, D Nickerson, J Ronsilvalli L (1999) Elementary Food Sci. 2nd ed. Ed. Avi Book. New York. 436 p.
Lee S, L Jeong D, H Young H (2001) Determination of seeding and harvesting time in snap bean. J. Korean 11:64-67.

Masaya P, J White W (1991) Adaptation to photoperiod and temperature. In: Common Beans. Reseach for Crop Improvement. A van Schoonhoven, O Voysest (eds). CAB Int. Centro Internacional de Agricultura Tropical. Colombia. pp:445-450.

Peixoto N, E Morales A, J Monteiro D (2001) Selection of climbing snap bean lines for the state of Goias. Hort. Brasil. 19:85-88.

Peixoto N, L Braz T, D Banzatto A, E Moraes A, F Moreira M (2002) Características agronómicas, productividade, qualidade de vagens e divergencia genetica em Feijoo-vegem de crecimiento indeterminado. Hort. Brasil. 20:447-451.

Quintana J M, H Harrison C, J Palta P, J Nienhuis, K Kmiecik (2001) Stomatal density and calcium concentration of six snap bean cultivars. J. Amer. Soc. Hort. Sci. 126:110-114.

Rosales S R, M Ochoa R, J A Acosta G (2001) Fenología y rendimiento del frijol en el antiplano de México y su respuesta al fotoperíodo Agrociencia 35:513-525.

Secretaría de Agricultura, Ganadería, Desarrollo Rural, Pesca y Alimentación SAGARPA (2003) Producción de Hortalizas. Disponible en http://www.sagarpa.gob.com.mx (2 de febrero de 2007).

Silbernagel M J, W Janssen, J H Davis C, G Montes de Oca (1991) Snap bean production in the tropics: Implications for genetic improvement. In: Common Bean: Reseach for Crop Improvement. A van Schoonhoven, O Voysest (eds). CAB Int Centro Internacional de Agricultura Tropical. pp:835-862.

Singh S P (1982) A key for identification of different growth habits y Phaseolus vulgaris L. Annu. Rep. Bean Improv. Coop. 25:92-95.

Snyder R L (1985) Hand calculating degree days. Agric. For. Meteor. 35:353-358.

Sosa P E (1979) Manual de Procedimientos Analíticos para Alimentos de Consumo Animal. Departamento de Zootecnia, Universidad Autónoma Chapingo, México. 115 p.

Steinbuch E (1980) The effect of heat shocks on quality retention of green beans during frozen storage. J. Food Technol. 15:353355.

Suzuki H H, T Tsukaguchi, Y Egawa (2001) Ultraestructural study on degeneration of tapetum in anther of snap bean (Phaseolus vulgaris L.) under heat stress. Sexual Plant Reprod. 13:293299. 